\title{
Research on the Problems and Countermeasures of Taiyuan Smart City Construction
}

\author{
Lifang Shi* \\ School of Political Science and Public Management, Shanxi University, China
}

Funding: This paper is a phased result of the project: Research on the Construction of Smart City in Shanxi Province in Big Data Era ( Project no.: 2017041033 - 4 ).

\begin{abstract}
Taking Taiyuan City as the research object, this paper sorts out the problems in smart city construction from four aspects, and based on the analysis of their deep-seated reasons, combined with the practice and development trend of Taiyuan City's smart city construction, 4 items have been put forward. Strong operability countermeasures and suggestions: strengthen the top-level design, clarify the construction ideas; formulate preferential investment policies to solve the capital dilemma; give full play to geographical advantages, clarify the focus of construction, etc.
\end{abstract}

Key words: Smart city; Digital economy; Transformation development; Livelihood services

Publication date: May, 2021; Publication online: 31 May, 2021

*Corresponding author: Lifang Shi, slf780430@163.com

Global economic integration, together with the wave of world urbanization and informatization, has promoted the development of cities in various aspects such as economy, society, and politics. At the same time, they have also been subjected to severe tests. In this context, "smart cities" have become a This strategy has been proposed and has become an irreversible trend for my country and even the world to solve urban development problems and achieve sustainable urban development. "Smart City" is the application of the Internet of Things, the Internet, cloud computing, and a new generation of information technologies that go beyond sensors, big data, and spatial geographic information integration systems, with high integration of smart technologies, high-end development of smart industries, and efficient and convenient services for the people A new urban operation model constructed for the main features.

As a "national smart city pilot" and a pioneering area for smart cities in Shanxi Province, Taiyuan City is building platforms represented by the "Smart Taiyuan" spatio-temporal information cloud platform, city management represented by digital city management, and the "Sky Eye" project as a representative Initial construction results have been achieved in the areas of livelihood services and other areas.

1 Problems in the construction of smart city in

\section{Taiyuan}

After decades of construction, Taiyuan's "smart city" has made great efforts to achieve smartness in energy, environmental protection, transportation, medical care, and urban management, with remarkable results. However, there are still some problems and challenges in the construction process, which need to be solved urgently.

\subsection{The linkage between city management and operation is not strong.}

At present, Taiyuan City has a relatively good foundation for management and operation, but the overall platform construction lags behind. Most of the departments are built separately and operate independently. The level of cross-departmental business collaboration is low, and there are "information islands" between departments. As a result, the construction of smart projects currently being carried out by various departments is fragmented.

\subsection{The pulling effect of the industry is not strong}


There is a shortage of high-end talents in Taiyuan, and there are not many high-level and high-educated talents in the inflow population. The stimulus for the development of the entire industry is not strong, and the dividends have not been effectively released. The information industry, especially the cloud computing, big data, and Internet of Things related to the construction of smart cities, has developed slowly, and has not been able to drive traditional industries.

\subsection{Insufficient innovation and lack of its own characteristics}

Taiyuan is an energy-heavy industrial city, and its ultimate goal is to build a green, low-carbon environmental protection model city. However, in the actual construction process, it did not work towards this goal. It just copied the construction experience of other cities mechanically, and did not form its own distinctive features, without its own characteristics.

\subsection{The promotion of citizen wisdom applications is not} high.

At present, the depth, breadth, and benefits of smart applications in Taiyuan City are not ideal, and some citizens have very weak awareness of smartness. According to the survey, only $3 \%$ of the population of "My Taiyuan" APP download behavior, and only $30 \%$ of them actually use this APP for water, electricity and heating payment and other application services. More people are Browsing local news.

\section{Analysis of the causes of problems in}

\section{Taiyuan's smart city construction}

\subsection{Lack of practical long-term planning}

In the early stage of building a smart city, Taiyuan did not have a reasonable planning plan and phased development goals, which did not provide good guidance for the development of smart cities, making the construction of some projects progress by leaps and bounds, and the construction of some projects stagnated. The reason is that the leadership role of Taiyuan's smart city construction organization has not been fully brought into play, and the government has not attached much importance to smart city construction.

\subsection{The smart city collaborative promotion mechanism has not yet been established}

In the construction of smart cities, there are bottlenecks such as ambiguity of responsibilities, insufficient synergy, low efficiency, and insufficient overall planning. The key lies in the inadequate innovation of the system and mechanism of smart city construction. Smart cities are a brand-new urban development concept and a brand-new urban operation form. Requires all government departments to use data thinking creatively, break down departmental interests and barriers, share information, and coordinate cooperation. It also requires the joint efforts of the government, enterprises and all walks of life to ensure and support.

\subsection{People's livelihood service capacity cannot meet construction needs.}

The improvement of people's livelihood services is the starting point for the construction of smart cities. It involves smart education, smart transportation, smart medical care, smart communities, smart culture, etc., which are closely related to life. The ultimate goal is to improve the quality of life of the public and improve the convenience of public life. Since Taiyuan does not have a unified urban data center planning and standards, most of the urban infrastructure libraries are currently in a state of separation, and some still have the problem of repeated construction. Moreover, due to the immature technology and operating mode of cloud computing, the construction and application of cloud platforms is still in its infancy, the actual application level is still relatively low, and the sharing between various cloud platforms is far from being realized.

\subsection{Shortage of professional organizations and talents}

A smart city is not accomplished overnight, but a complex and huge system project. Errors and problems in any link will affect the entire construction cycle. This requires professional organizations and personnel to track and guide at any time to solve problems in a timely manner. Although Taiyuan City has established a smart city construction leadership group headed by the mayor, it is not a professional organization, and the decision-making is unscientific and the implementation is not in place, which greatly reduces the scientific and rationality of smart city construction. Sex and efficiency.

\subsection{Lack of funds needed for smart cities}

According to the experience of "smart cities" already under construction, it is difficult to successfully build smart cities without sufficient capital investment. In recent years, the economy of Taiyuan City, which is based on Energy and Heavy Industries, has faced more severe challenges. There are more fiscal revenues and expenditures, less revenue, 
limited disposable fiscal revenues, narrow financing channels, and the funds used for smart city construction are decreasing year by year. Intensified the difficulty of smart city construction.

\section{Proposals for perfecting the construction of}

\section{Taiyuan's smart city}

\subsection{Strengthen the top-level design and clarify the construction ideas.}

In the planning and construction of smart cities, we must strive not to follow the trend and blindly follow the trend, and to fully integrate the actual conditions of the region to build a new model of sustainable development of smart cities that suits the characteristics of each region.

3.1.1 Strengthen top-level planning and coordinate construction.

Promote Taiyuan's industrial upgrading and structural adjustment with new technologies and new thinking, establish a new development model with more vitality and potential, and at the same time follow the objective laws and national standards of smart city construction, and use our rich experience in smart city planning to follow technology The law of evolution, the establishment of a smart city expert research group, to provide intellectual support for major project decision-making. Fully understand that the construction of a smart city is a long-term and arduous major project, pay attention to overall planning and overall coordination, and coordinate advancement in a planned and hierarchical manner.

\subsubsection{Government guidance and market operation}

Give full play to the decisive role of the market, and earnestly bring into play the role of new smart city construction in promoting economic and social development, improving services to the people's livelihood, and improving people's quality of life, and use the application effect as the main indicator to measure the construction effectiveness. Gradually introduce diversified social investment in smart application projects involving people's livelihood to make up for the lack of government financial funds, reduce smart city operating costs, achieve open cooperation, and build a win-win situation.

3.1.3 Put the foundation first and lay the framework.

Complete the government cloud platform construction project as soon as possible, and gradually shift the focus of e-government work from the construction and improvement of infrastructure to the focus on promoting the construction of a security system and data sharing and exchange. Use big data and "Internet + " thinking to promote the adjustment and transformation of urban governance models and economic structures, innovate governance decision-making models, enhance the initiative, accuracy and convenience of urban management and services, and establish new business models, business models and consumption mode.

\subsubsection{Resource sharing and clear ownership}

Promote the data integration of different industries, systems, and departments, promote the interconnection and interoperability of business systems, and further clarify the possession and use rights of data resources, establish a basic structure of data ownership and use rights, and design corresponding restriction mechanisms. Avoid problems such as duplication of construction, development, fighting each other, and fragmentation that may arise in the construction process. At the same time, it is necessary to establish a number of demonstration projects based on the actual development of the city to promote the construction of application systems in key economic and social fields.

\subsection{Improve the organizational structure and establish a departmental linkage mechanism.}

Improve the work leadership team with the main leaders of the municipal government as the team leader and the relevant departments as the team members to undertake various coordination tasks for the construction of smart cities.

\subsubsection{Strengthen target assessment.}

Study the establishment of scientific and reasonable smart city construction goals, and establish a smart city construction evaluation and assessment mechanism. Decompose the construction goals layer by layer and implement them to various departments, regularly organize assessment and assessment of smart city construction and release assessment results, and use the assessment results as the basis for the performance evaluation of government departments at all levels and the assessment of rewards and punishments for leading cadres to ensure smart city projects The construction achieved the expected results.

\subsubsection{Strengthen the training of relevant departments.}

The construction of a smart city is a complex and huge project that cannot be completed overnight. This requires the continuous improvement of the quality of relevant business departments and the leadership's awareness and attention to the construction of smart cities. By opening training courses 
and hiring relevant experts to explain professional knowledge, problems and doubts existing in the current construction process can be resolved. At the same time, by strengthening publicity, encourage the whole society to join the enthusiasm for smart city construction and create a good atmosphere.

\subsubsection{Establish a working mechanism for departmental} coordination and linkage.

Construct a promotion system that is led by the government, with enterprises and citizens as the main body, market-oriented, and a combination of production, education, research and application. Unify the ideology of all responsible units, enhance the overall awareness and sense of responsibility of all departments, establish a work system where the leaders in charge are specifically responsible, the division of responsibilities is clear, the departments actively and closely cooperate, and complete tasks on time and according to requirements, and promote the construction of smart cities in a planned and step-by-step manner Work to ensure the smooth development of smart city construction.

\subsection{Formulate preferential investment policies to solve funding difficulties.}

3.3.1 Reasonably choose the investment and financing model.

From the construction of infrastructure, to the interconnection of information between various systems, the upgrade, operation, and maintenance of the city after the completion of a smart city requires a large amount of capital investment, which requires a high level of urban economic development. Taiyuan City can guide social funds to participate in smart city construction through franchising and purchasing services, and encourage qualified companies to issue corporate bonds to raise funds for smart city construction, such as the PPP model.

\subsubsection{Formulate preferential taxation and investment} policies.

Various projects for smart city construction require a large amount of funds, and the investment return cycle is long. Relying solely on credit from financial institutions is bound to increase the operating costs and liabilities of enterprises. If the government's industry guidance fund takes the lead, formulate preferential tax and investment policies, encourage and guide individuals, enterprises and social organizations with investment intentions to invest in smart city projects and smart industries in the form of direct investment, equity participation, etc., and enrich their financing channel.

\subsection{Give full play to geographical advantages and clarify the focus of construction.}

Domestic and foreign experience in the construction of smart cities has shown that the effect of full bloom in the construction of smart cities is not obvious. It must reflect its own characteristics and rely on local superior resources to carry out focused construction.

\subsubsection{Build a cloud computing integrated computing center.}

Establish an operating model of "government building and enterprises taking the stage", strengthen the application of cloud computing in smart markets and smart government affairs, and promote the establishment of Internet data processing centers by communication operating companies.

3.4.2 Improve the construction of urban basic data resources.

A true smart city should include various aspects such as public service management, market economy organization, and citizens' living needs. Therefore, we need to build on our own big data resources and integrate information service data in many fields such as economic society, market development, and residents' lives to promote the construction of smart cities.

\subsubsection{Construct a data interoperability carrier.}

Promote the unity of data management between multiple fields and organizations, and on this basis, vigorously support the construction of urban data interoperability carriers. Form a government service management sharing and coordination system mechanism with data sharing standards and interfaces, and realize information sharing and business coordination between government departments.

\subsubsection{Data management and operation carrier construction}

Based on the optimization and improvement of multiple databases and data resources, and establish a consistent standard data management service system. Public release through the data release platform to promote enterprises, scientific research institutions, commercial service units, etc. to provide continuous impetus for the development of urban people, enterprises, and industries, and promote the development of emerging industries in the city.

3.4.5 Full coverage of smart city communication infrastructure construction

The first is to accelerate the construction of the city's data system and build a fiber-optic city. The second is to promote 
the full coverage of urban $5 \mathrm{G}$ networks. Integrate the data resources of various private networks and general networks, continuously expand the service boundary of Internet government affairs, form an "Internet +" government service mechanism managed by "one network", and promote the full use of e-government service efficiency. Construct a wireless network infrastructure with strong levels, wide coverage, and sufficient service effects, and promote the wirelessization of the urban Internet.

\subsubsection{Speed up the construction of urban smart infrastructure.}

It is necessary to integrate the information and data resources of various underground infrastructure constructions in the city, such as power supply, water supply, urban heating services, optical cables and other resources. Such integrated resources are the basis for the development of a smart city, and can effectively improve the service level and management level of the smart city.

\subsection{Vigorously implement the strategy of bringing in and going out.}

The construction of a smart city must focus on long-term development. Technicians cannot only rely on the introduction from outside the region. They can cultivate and produce results on their own to truly serve the long-term construction. The problems encountered at the technical level can be realized through the cooperation of government, school, and enterprise, led by the government; through the integration of the school's faculty, more attractive conditions are opened, such as student settlement, teaching investment, employment guidance, etc. Ways to create convenient conditions for the school; the enterprise invests funds to allow the school's scientific research achievements and outstanding talents to land in the enterprise. Through the orderly cooperation of the three parties, a win-win situation will be created.

\subsubsection{Implement the introduction strategy.}

Establish a complete professional talent team, and through the talent "introduction" strategy to attract more high-end compound talents who understand technology and management. In order to further stabilize the professional talent team, it is necessary to provide a multi-faceted guarantee for talents from the aspects of system and mechanism. This guarantee is not only material incentives, but also spiritual encouragement. It is necessary to establish a number of preferential development policies for Only talented people can configure a good atmosphere.

\subsubsection{Implement the strategy of going out.}

By strengthening "city cooperation", let young cadres or talents in professional fields learn to build better cities intelligently, or ask the other party to send an expert group for guidance to realize talent sharing.

\section{Disclosure statement}

The author declares no conflict of interest.

\section{References}

[1] Huang C, Peng CC, Su J. Smart governance [M]. Beijing: Tsinghua University Press, 2017.

[2] Green Ben. The Smart Enough City, [M]. New York: The MIT Press, 2019.

[3] Liu J, Chen BS. Public dialogue policy implementation: an important breakthrough for building a service-oriented government [J]. Chinese Administration, 2013, (05): 34-38.

[4] Yu SY, Yang DL. Smart government portal based on big data: from idea to practice[J]. E-government, 2013, (02): 65-74.

[5] Guo XR, Wu XF. Research on the construction model of smart city[J]. Journal of Surveying and Mapping Science and Technology, 2013, (3): 319-323.

[6] Sun XX, Smart City Management: A New Exploration of City Management towards Smart City-Taking Hangzhou as an example [J]. City Management and Technology, 2013, (05): 61-63. 